\title{
Minimal covers of the Archimedean tilings, part II
}

\author{
Mark Mixer \\ Department of Mathematics and Statistics \\ Williams College \\ Williamstown, MA, USA \\ mark.mixer@williams.edu
}

\author{
Daniel Pellicer* \\ Research Center on Mathematical Sciences \\ National University of Mexico \\ Morelia, Michoacan, Mexico \\ pellicer@matmor .unam.mx
}

\author{
Gordon Williams ${ }^{\dagger}$ \\ Department of Mathematics and Statistics \\ University of Alaska Fairbanks \\ Fairbanks, Alaska, USA \\ gwilliams@alaska.edu
}

Submitted: Jan 24, 2013; Accepted: Apr 12, 2013; Published: Apr 24, 2013

Mathematics Subject Classifications: 52B15, 52B70, 52C20

\begin{abstract}
In Part I of this paper ([PW12]), the minimal regular covers of three of the eight Archimedean tilings were determined. However, the computations described in that work grow more complicated as the number of flag orbits of the tilings increases. In Part II, we develop a new technique in order to present the minimal regular covers of certain periodic abstract polytopes. We then use that technique to finish determining the minimal regular covers of the Archimedean tilings.
\end{abstract}

\section{Introduction}

Symmetric maps on surfaces, and their automorphism groups, have been studied since the early 20th century, mainly in the case when the maps are finite, that is, when they lie on compact surfaces (see [Bra27], [CM80]). Much is known about the regular covers and the closely related monodromy groups of such finite maps (e.g., [Orb07, Wil76, Wil94, Wil02]). However, tilings of the Euclidean or hyperbolic plane are examples of maps on noncompact surfaces, and as such, require somewhat different methods of investigation.

\footnotetext{
*partially supported by PAPIIT-Mexico under grant IB100312 and CONACYT project 166951

${ }^{\dagger}$ partially supported by PAPIIT-Mexico under grant IB100312

All of the authors would also like to acknowledge the Fields Institute's 2011 Workshop on Symmetry in Graphs, Maps and Polytopes.
} 
Since the late 20th century, maps whose vertex-figures are polygons have been studied as abstract polyhedra (rank 3 abstract polytopes) [MS02, Section 6B]. Much work has been done to understand (abstract) regular polytopes, where [MS02] is the primary reference work. However, in comparison, much less is known about polytopes with less symmetry. A particularly useful tool for the study of non-regular abstract polytopes is [Har99a], in which Hartley demonstrates that any abstract polytope can be realized as the quotient of a regular (abstract) polytope. There is a strong connection between this idea and the concept of regular covers of maps. In particular, every map has a minimal regular cover, in the sense that no other regular cover is covered by the minimal one. It is easy to demonstrate that the minimal regular cover of a polyhedron is itself a polyhedron (details are provided in $[\mathrm{MPW}]$ ). This paper and its predecessor [PW12] are part of a project whose goal is to better understand the structure of quotients and covers both geometrically and algebraically. For a discussion of topological aspects of the covers of infinite maps see $[\mathrm{CPR}+]$.

Symmetric tilings have been the subject of intense interest and investigation, especially since the publication of Grübaum and Shephard's Tilings and Patterns [GS87]. Consequently, the Archimedean tilings provide a natural starting point in the study of regular covers of infinite maps. In [PW11b] representations of the Archimedean tilings as quotients of regular hyperbolic tilings were provided by means of a general description developed by Hartley in [Har99a] (more basic information about such representations is available in [Har99b] and [HW10]). The minimal regular covers of the three Archimedean tilings (3.6.3.6), (4.8.8) and (3.12.12) were found in [PW12]. These tilings have in common that the number of orbits on flags under the symmetry group is at most 3 . The technique used consists of finding the intersection of stabilizers of certain flags under the action of the monodromy group of the tiling. While this method is very general, and can be used to work with arbitrary abstract polytopes, the calculations were lengthy and seemed intractable for the remaining five Archimedean tilings.

Using new techniques, in this paper we complete the determination of the minimal regular covers of the Archimedean tilings. The theory leading to the procedure which we used to find the minimal regular covers of the Archimedean tilings (3.4.6.4), (3.3.4.3.4), (3.3.3.4.4), (4.6.12) and (3.3.3.3.6) is provided in Section 3. This procedure is a natural tool which could be applied to other periodic tessellations of Euclidean spaces. In Section 4 we show the details of this procedure while considering the tiling (3.4.6.4). Finally, an application of this procedure to finite maps on the torus is discussed in Section 5.

\section{Basic notions and previous results}

In this paper, we define polyhedra as abstract polytopes of rank 3 in the sense of [MS02]; that is, a polyhedron is a partially ordered set with a rank function on the set $\{0,1,2\}$

satisfying certain properties. Following the notation of convex polyhedra, the vertices, edges and faces of an abstract polyhedron are its elements of rank 0,1 and 2 respectively. Every vertex belongs to at least two edges, and every face contains at least two edges.

A section $F / G$ of a polyhedron is defined when $G \leqslant F$ to be the partially ordered 
set of all elements $H$ such that $G \leqslant H \leqslant F$, that is $F / G:=\{H \in \mathcal{P} \mid G \leqslant H \leqslant F\}$. The vertex-figure at a vertex $v$ is the section $\{F \in \mathcal{P} \mid v \leqslant F\}$. Abstract polyhedra satisfy the diamond condition, that is, every edge contains precisely two vertices and is contained in precisely two faces, and the section determined by an incident face $f$ and vertex $v$ pair contains exactly two edges. A flag is a (vertex, edge, face) triple of mutually incident elements of the partially ordered set. As a consequence of the diamond condition, given $i \in\{0,1,2\}$ and a flag $\Psi$, there exists a unique flag $\Psi^{i}$ that coincides with $\Psi$ in all elements except in that of rank $i$. The flag $\Psi^{i}$ is called the $i$-adjacent flag of $\Psi$. Finally, an abstract polyhedron is also strongly flag-connected, that is, any two flags in a polyhedron can be connected by a sequence of adjacent flags, and this connectivity is preserved when restricted to either faces or vertex-figures.

The degree of a vertex $v$ is the number of edges containing $v$, and the co-degree of a face $f$ is the number of edges contained in $f$. Whenever every vertex of a polyhedron $\mathcal{P}$ has the same degree $q$, and every face of $\mathcal{P}$ has the same co-degree $p$ we say that $\mathcal{P}$ is equivelar and has Schläfli type $\{p, q\}$.

An automorphism of a polyhedron $\mathcal{P}$ is an order preserving bijection of its elements. We say that a polyhedron is regular if its automorphism group $\Gamma(\mathcal{P})$ acts transitively on the set of flags of $\mathcal{P}$. The Platonic solids and the familiar regular tessellations of the Euclidean plane by triangles, squares, or hexagons $\left(3^{6}, 4^{4}\right.$, and $6^{3}$ in the notation of [GS87]) are examples of abstract regular polyhedra.

A string $C$-group $G$ of rank 3 is a group which has distinct distinguished involutory generators $\rho_{0}, \rho_{1}, \rho_{2}$ satisfying that $\left(\rho_{0} \rho_{2}\right)^{2}=i d$, the identity in $G$, and that $\left\langle\rho_{0}, \rho_{1}\right\rangle \cap$ $\left\langle\rho_{1}, \rho_{2}\right\rangle=\left\langle\rho_{1}\right\rangle$ (the latter is called the intersection condition). The automorphism group of an abstract regular polyhedron $\mathcal{P}$ is always a string C-group of rank 3. Given an arbitrarily chosen base flag $\Phi$ of $\mathcal{P}, \rho_{i}$ can be taken to be the (unique) automorphism mapping $\Phi$ to the $i$-adjacent flag $\Phi^{i}$. Furthermore, any string $\mathrm{C}$-group $\Gamma$ of rank 3 is the automorphism group of an abstract regular polyhedron $\mathcal{P}(\Gamma)$ [MS02, 2B], so, up to isomorphism, there is a one-to-one correspondence between the string C-groups of rank 3 and the abstract regular polyhedra. Thus, in the study of regular abstract polyhedra we may either work with the polyhedron as a poset or with its automorphism group.

Examples of regular polyhedra are the regular tilings. We define a tiling of the (spherical, Euclidean or hyperbolic) plane as an edge to edge covering of the plane by convex polygons whose interiors are pairwise disjoint. There are three regular tilings of the Euclidean plane, namely the tessellations by equilateral triangles, squares and regular hexagons.

For any polyhedron $\mathcal{P}$ we define permutations $r_{0}, r_{1}, r_{2}$ on $\mathcal{F}(\mathcal{P})$ by

$$
\Psi r_{i}:=\Psi^{i}
$$

for every flag $\Psi$ of $\mathcal{P}$ and $i=0,1,2$ (note that these are not automorphisms of $\mathcal{P}$ ). The group $\operatorname{Mon}(P):=\left\langle r_{0}, r_{1}, r_{2}\right\rangle$ will be referred to as the monodromy group of $\mathcal{P}$ [HOW09]. It is now well known that $\operatorname{Mon}(\mathcal{P})$ is a string C-group for every polyhedron $\mathcal{P}$ (see for example [MPW]). 
Note that, by definition of automorphism, the action of each $r_{i}$ commutes with the automorphisms of any given polyhedron. That is,

$$
\alpha\left(\Psi r_{i}\right)=(\alpha \Psi) r_{i}
$$

for $i=0,1,2$ and $\alpha \in \Gamma(\mathcal{P})$. More generally, an inductive process shows that the action of the monodromy group on the flags commutes with the action by automorphisms, that is,

$$
\alpha(\Psi w)=(\alpha \Psi) w
$$

for every $w \in \operatorname{Mon}(\mathcal{P}), \alpha \in \Gamma(\mathcal{P})$. In other words, the left action of $\Gamma(\mathcal{P})$ and right action of $\operatorname{Mon}(\mathcal{P})$ on the set of flags are compatible.

The flag action of a string C-group $\Gamma=\left\langle\rho_{0}, \rho_{1}, \rho_{2}\right\rangle$ on $\mathcal{P}$ is the group homomorphism $\Gamma \rightarrow \operatorname{Mon}(P)$ defined by $\rho_{i} \mapsto r_{i}$, provided such a homomorphism exists. We say that the regular polyhedron $\mathcal{P}$ is a cover of $\mathcal{Q}$, denoted by $\mathcal{P} \searrow \mathcal{Q}$, if $\mathcal{Q}$ admits a flag action from $\Gamma(\mathcal{P})$. For example, the (universal) polyhedron with automorphism group isomorphic to the Coxeter group $[\infty, \infty]:=\left\langle\rho_{0}, \rho_{1}, \rho_{2} \mid\left(\rho_{0} \rho_{2}\right)^{2}=i d\right\rangle$ covers all other polyhedra. Whenever the least common multiple of the co-degrees of the faces of a polyhedron $\mathcal{P}$ is $p$, and the least common multiple of the vertex degrees of $\mathcal{P}$ is $q, \mathcal{P}$ is covered by the tiling $\{p, q\}$ whose automorphism group is isomorphic to the string Coxeter group

$$
[p, q]:=\left\langle\rho_{0}, \rho_{1}, \rho_{2} \mid\left(\rho_{0} \rho_{2}\right)^{2}=\left(\rho_{0} \rho_{1}\right)^{p}=\left(\rho_{1} \rho_{2}\right)^{q}=i d\right\rangle .
$$

Recall that $\{p, q\}$ can be viewed as a regular tiling of the sphere, Euclidean plane or hyperbolic plane, depending on whether $\frac{1}{p}+\frac{1}{q}$ is bigger than, equal to, or less than $\frac{1}{2}$, respectively.

Whenever $\mathcal{P} \searrow \mathcal{Q}$, the polyhedron $\mathcal{Q}$ is totally determined by $\mathcal{P}$ and the stabilizer $N$ of a chosen base flag $\Phi$ of $\mathcal{Q}$ under the flag action of $\Gamma(\mathcal{P})$. Indeed, $\mathcal{Q}=\mathcal{P} / N$, the polytope whose faces are orbits under the action of $N$ on $\mathcal{P}$ [Har99a].

Since the monodromy group of every polyhedron is a string C-group, any polyhedron $\mathcal{Q}$ is automatically equipped with a regular cover, $\mathcal{P}(\operatorname{Mon}(\mathcal{Q}))$. Moreover, it is straightforward to demonstrate that

$$
\operatorname{Mon}(Q) \cong \Gamma / \operatorname{Core}(\Gamma, N)
$$

where $\Gamma$ is the automorphism group of any regular cover of $\mathcal{Q}, N$ is the stabilizer of a flag in $\mathcal{Q}$ under the flag action of $\Gamma$, and the core, $\operatorname{Core}(\Gamma, N)$, is the largest normal subgroup of $\Gamma$ in $N$ (see [MPW] for details). In particular, it follows from (3) that there is a group homomorphism from $\Gamma$ to $\operatorname{Mon}(\mathcal{Q})$ mapping the generator $\rho_{i}$ to $r_{i}$, implying that any regular cover of $\mathcal{Q}$ also covers the polyhedron $\mathcal{P}(\operatorname{Mon}(\mathcal{Q}))$. This motivates the name "minimal regular cover of $\mathcal{Q}$ " for $\mathcal{P}(\operatorname{Mon}(\mathcal{Q}))$ since it is actually the smallest possible regular cover.

As a consequence of the previous discussion, the monodromy group of a regular polytope $\mathcal{P}$ is isomorphic to $\Gamma(\mathcal{P}) / \operatorname{Core}(\Gamma, N)$, where $N$ is the stabilizer of any flag in $\mathcal{P}$. Note that in this case $\operatorname{Core}(\Gamma, N)$ is trivial, since the stabilizer of all flags is the same as the 
stabilizer of any individual flag (due to regularity) and every conjugate of the stabilizer $N$ of a flag of $\mathcal{P}$ is the stabilizer of another flag of $\mathcal{P}$. Hence $\Gamma(\mathcal{P}) \cong \operatorname{Mon}(P)$ (for further details of this fact see [MPW]).

This leads to a useful reinterpretation of the condition for a regular polyhedron $\mathcal{P}$ to be a cover of $\mathcal{Q}$. The fact that $\mathcal{Q}$ admits a flag action by $\Gamma(\mathcal{P})$ is equivalent to there being an epimorphism from $\operatorname{Mon}(P)$ to $\operatorname{Mon}(Q)$. Thus, we find it more natural to understand the cover $\mathcal{P} \searrow \mathcal{Q}$ as an epimorphism of monodromy groups, instead of as a homomorphism from an automorphism group to a monodromy group. This perspective is motivated by the natural way in which $i$-adjacent flags of $\mathcal{P}$ are mapped into $i$-adjacent flags of $\mathcal{Q}$. Henceforth we shall proceed according to this notion and use the generators $r_{0}, r_{1}, r_{2}$ of $\operatorname{Mon}(P)$ instead of those of $\Gamma(\mathcal{P})$ to denote the action on the flags of $\mathcal{Q}$.

Several geometric objects can be viewed as abstract polyhedra, for example the convex polyhedra and the plane tilings. In such cases, the symmetry group is the group of isometries of the ambient space preserving the polyhedron. Every symmetry can be understood as an automorphism of the abstract object, but in general not every automorphism can be realized as a symmetry of a geometric realization of the abstract object. Throughout the remainder of this paper we will use the term "polyhedra" to mean either the geometric objects or abstract polyhedra, as appropriate.

An Archimedean or uniform tiling of the Euclidean plane is a non-regular tiling by regular convex polygons such that its automorphism group acts transitively on the vertices. There are eight Archimedean tilings, determined by the types and cyclic order of the regular polygons meeting at each vertex. Following [GS87], we denote each Archimedean tiling by a symbol of the form $\left(p_{1} \cdot p_{2} \ldots \ldots p_{k}\right)$ where each $p_{i}$ indicates a $p_{i}$-gon in a cyclic ordering of the faces around a vertex. Thus (3.6.3.6) denotes the Archimedean tiling of the plane in which each vertex is surrounded by a triangle, a hexagon, a triangle and a hexagon (or any cyclic reordering or reversal of this sequence).

Every Archimedean tiling of the plane contains faces of different sizes. In some of these tilings each flag orbit $\mathcal{O}$, under the symmetry group, is determined by the size of the faces in flags in $\mathcal{O}$ together with the size of the faces of their 2-adjacent flags, thus giving a combinatorial description of the flag orbits. More generally, each flag orbit $\mathcal{O}$, under the symmetry group of an Archimedean tiling of the plane, is determined by the sizes of the faces in flags in $\mathcal{O}$ together with the sizes of the faces in images of the flags in $\mathcal{O}$ by some monodromy elements which depend only on the tiling. This combinatorial description of the flag orbits under the symmetry group implies that the symmetry group and the automorphism group of any Archimedean tiling are isomorphic. Whenever $\mathcal{A}$ is an Archimedean tiling we can abuse notation and understand the elements of $\Gamma(\mathcal{A})$ either as combinatorial automorphisms or as symmetries of the geometric tessellation as needed. The eight Archimedean tilings are listed in Table 1.

A toroidal quotient of a tiling $\mathcal{T}$ of $\mathbb{E}^{2}$ is a quotient $\mathcal{T} /\left\langle t_{1}, t_{2}\right\rangle$ where $t_{1}$ and $t_{2}$ are two translations with respect to linearly independent vectors that are also symmetries of $\mathcal{T}$. The vertices, edges and faces of $\mathcal{T} /\left\langle t_{1}, t_{2}\right\rangle$ are the orbits of the vertices, edges and faces of $\mathcal{T}$ under the action of $\left\langle t_{1}, t_{2}\right\rangle$, and the incidence in $\mathcal{T} /\left\langle t_{1}, t_{2}\right\rangle$ is the natural incidence induced from that in $\mathcal{T}$. 


\section{Structure of the covers}

In this section we show that the monodromy group of every Archimedean tiling $\mathcal{A}$ is a finite extension of a free abelian group of finite rank. As we shall see, the rank of such an abelian group is $2 k$, where $k$ is the number of flag orbits of $\mathcal{A}$ under $\Gamma(\mathcal{A})$. Specific details of the monodromy groups of the Archimedean tilings are given in Lemmas 1 to 6 . The main result is stated as Theorem 7 .

Lemma 1. Let $\mathcal{A}$ be an Archimedean tiling of $\mathbb{E}^{2}$. There exists an orbit $\mathcal{O}$ of flags under the action of $\Gamma(\mathcal{A})$, such that there exist $z_{\mathcal{O}}, w_{\mathcal{O}} \in M o n(\mathcal{A})$ fixing all flags of $\mathcal{A}$ not in $\mathcal{O}$ while acting on flags in $\mathcal{O}$ like translational symmetries of $\mathcal{A}$ in two linearly independent directions.

Proof. In Table 1 we show an element $z_{\mathcal{O}}$ for each of the choices of $\mathcal{A}$. In each case, the orbit $\mathcal{O}$ is represented by the flag in gray in Figure 1. For each tessellation, the translation vector is with respect to the basis indicated in the same figure, where the white-head arrow indicates the first vector of the basis.

\begin{tabular}{|c|c|c|}
\hline $\mathcal{A}$ & $z_{\mathcal{O}}$ & Translation vector \\
\hline$(3.6 .3 .6)$ & {$\left[\left(r_{0} r_{1}\right)^{3} r_{2} r_{1} r_{2}\right]^{2}$} & $(2,0)$ \\
\hline$(4.8 .8)$ & {$\left[\left(r_{0} r_{1}\right)^{4} r_{2}\right]^{4}$} & $(4,0)$ \\
\hline$(3.12 .12)$ & {$\left[\left(r_{0} r_{1}\right)^{3} r_{2}\right]^{3}\left[\left(r_{1} r_{0}\right)^{3} r_{2}\right]^{3}$} & $(2,0)$ \\
\hline$(3.4 .6 .4)$ & $\left(r_{0} r_{1}\right)^{3} r_{2}\left(r_{0} r_{1}\right)^{6} r_{2}\left(r_{1} r_{0}\right)^{3} r_{2}\left(r_{0} r_{1}\right)^{6} r_{2}$ & $(2,0)$ \\
\hline$(3.3 .4 .3 .4)$ & {$\left[\left(r_{1} r_{0}\right)^{3} r_{2} r_{1} r_{2}\right]^{2}\left[\left(r_{0} r_{1}\right)^{3} r_{2} r_{1} r_{2}\right]^{2}$} & $(1,1)$ \\
\hline$(3.3 .3 .4 .4)$ & {$\left[\left(r_{0} r_{1}\right)^{3} r_{2}\left(r_{0} r_{1}\right)^{2} r_{2} r_{0} r_{1} r_{2}\left(r_{1} r_{0}\right)^{2} r_{2} r_{1} r_{2} r_{1} r_{2}\right]^{2}$} & $(2,0)$ \\
\hline$(4.6 .12)$ & $\left(r_{0} r_{1}\right)^{4}\left[r_{2}\left(r_{0} r_{1}\right)^{-4}\right]^{2} r_{2}\left(r_{0} r_{1}\right)^{6} r_{2}\left[\left(r_{0} r_{1}\right)^{4} r_{2}\right]^{2}\left(r_{0} r_{1}\right)^{-4} r_{2}\left(r_{0} r_{1}\right)^{6} r_{2}$ & $(2,2)$ \\
\hline$(3.3 .3 .3 .6)$ & {$\left[\left(r_{0} r_{1}\right)^{3} r_{2} r_{1} r_{2} r_{0} r_{1} r_{2}\right]^{4}$} & $(4,0)$ \\
\hline
\end{tabular}

Table 1: Element $z_{\mathcal{O}} \in \operatorname{Mon}(A)$

In all cases we can obtain $w_{\mathcal{O}}$ by conjugating $z_{\mathcal{O}}$ with an element in $\operatorname{Mon}(\mathcal{A})$ fixing orbit $\mathcal{O}$. For instance

$$
\begin{aligned}
(3.6 .3 .6) & \rightarrow w_{\mathcal{O}}=r_{1} r_{0} z_{\mathcal{O}} r_{0} r_{1} \\
(4.8 .8) & \rightarrow w_{\mathcal{O}}=r_{1} r_{0} r_{1} z_{\mathcal{O}} r_{1} r_{0} r_{1}, \\
(3.12 .12) & \rightarrow w_{\mathcal{O}}=r_{1} r_{0} r_{1} z_{\mathcal{O}} r_{1} r_{0} r_{1} \\
(3.4 .6 .4) & \rightarrow w_{\mathcal{O}}=r_{0} r_{1} z_{\mathcal{O}} r_{1} r_{0} \\
(3.3 .4 .3 .4) & \rightarrow w_{\mathcal{O}}=r_{1} r_{0} z_{\mathcal{O}} r_{0} r_{1} \\
(3.3 .3 .4 .4) & \rightarrow w_{\mathcal{O}}=r_{2} r_{1} r_{0} r_{1} r_{2} z_{\mathcal{O}} r_{2} r_{1} r_{0} r_{1} r_{2}, \\
(4.6 .12) & \rightarrow w_{\mathcal{O}}=r_{1} r_{0} r_{1} z_{\mathcal{O}} r_{1} r_{0} r_{1} \\
(3.3 .3 .3 .6) & \rightarrow w_{\mathcal{O}}=r_{1} r_{0} z_{\mathcal{O}} r_{0} r_{1} .
\end{aligned}
$$




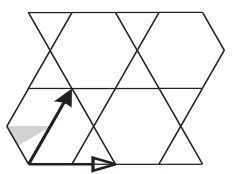

(3.6.3.6)

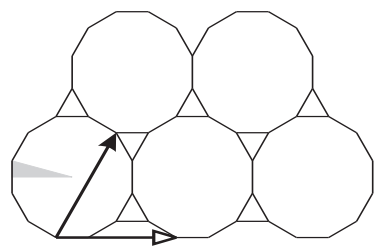

(3.12.12)

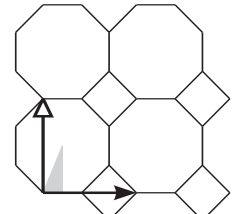

(4.8.8)

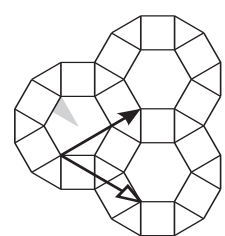

(3.4.6.4)

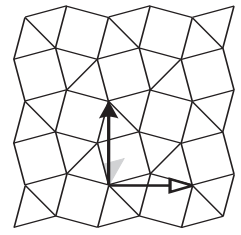

(3.3.4.3.4)

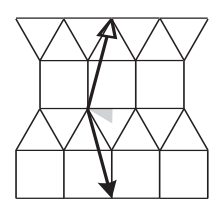

(3.3.3.4.4)

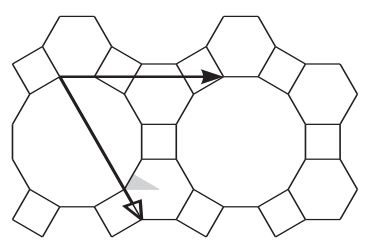

(4.6.12)

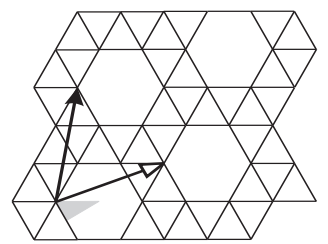

(3.3.3.3.6)

Figure 1: Bases for the translation subgroups of the symmetry groups of the Archimedean tilings.

Lemma 2. Let $\mathcal{A}$ be an Archimedean tiling of $\mathbb{E}^{2}$ and let $\mathcal{O}_{1}, \ldots, \mathcal{O}_{k}$ be its flag orbits under $\Gamma(\mathcal{A})$. Then, for each $j \in\{1, \ldots, k\}$ there exist $z_{j}, w_{j} \in \operatorname{Mon}(\mathcal{A})$ fixing all flags of $\mathcal{A}$ not in $\mathcal{O}_{j}$ while acting on flags in $\mathcal{O}$ like translational symmetries of $\mathcal{A}$ in two linearly independent directions.

Proof. Without loss of generality assume that $z_{1}$ and $w_{1}$ are respectively $z_{\mathcal{O}}$ and $w_{\mathcal{O}}$ as in Lemma 1. Recall that the action of the monodromy group is transitive on the flags of $\mathcal{A}$. This implies that if a flag on orbit $\mathcal{O}_{1}$ is reached from a flag $\Phi$ on orbit $\mathcal{O}_{j}$ by the element $f \in \operatorname{Mon}(\mathcal{A})$ then we may define $z_{j}$ and $w_{j}$ as $f z_{1} f^{-1}$ and $f w_{1} f^{-1}$ respectively. To see that $z_{j}$ and $w_{j}$ act on flags in $\mathcal{O}_{j}$ like translational symmetries of $\mathcal{A}$ in two linearly independent directions, it suffices to consider the corresponding action on the triangle of the barycentric subdivision of the tiling $\mathcal{A}$ corresponding to $\Phi$ and the triangle corresponding to $\Phi f$. Thus the geometric effect of $f z_{1} f^{-1}$ on $\Phi$ is the translation that maps $\Phi f$ to $\Phi f z_{1}$.

In the following three lemmas we concentrate our attention on properties of the group $\left\langle z_{j}, w_{j} \mid j \in\{1, \ldots, k\}\right\rangle$.

Lemma 3. Let $\mathcal{A}, z_{j}$, and $w_{j}$ be as in Lemma 2. Then $\left\langle z_{j}, w_{j} \mid j \in\{1, \ldots, k\}\right\rangle \cong \mathbb{Z}^{2 k}$.

Proof. By definition, an element in $\operatorname{Mon}(\mathcal{A})$ equals $i d$ if and only if it acts like $i d$ on all flags of $\mathcal{A}$. Note that the elements $z_{i} z_{j} z_{i}^{-1} z_{j}^{-1}, w_{i} w_{j} w_{i}^{-1} w_{j}^{-1}$ and $z_{i} w_{j} z_{i}^{-1} w_{j}^{-1}$ act 
on all flags of $\mathcal{A}$ like a composition of two (maybe trivial) translational symmetries of $\mathcal{A}$ and their inverses. Since the action of any pair of translations commute, $z_{i} z_{j} z_{i}^{-1} z_{j}^{-1}$, $w_{i} w_{j} w_{i}^{-1} w_{j}^{-1}$ and $z_{i} w_{j} z_{i}^{-1} w_{j}^{-1}$ act like $i d$ on all flags, implying that every pair of generators of $\left\langle z_{j}, w_{j} \mid j \in\{1, \ldots, k\}\right\rangle$ must commute. Clearly $z_{i}$ and $w_{i}$ have infinite order, and Lemma 2 implies that none of these generators can be expressed in terms of the others. Thus the lemma holds.

Lemma 4. Let $\mathcal{A}, z_{j}$, and $w_{j}$ be as in Lemma 2. Then $\left\langle z_{j}, w_{j} \mid j \in\{1, \ldots, k\}\right\rangle$ has finite index in $\operatorname{Mon}(\mathcal{A})$.

Proof. Let $t_{1}$ and $t_{2}$ be the translations in $\Gamma(\mathcal{A})$ such that if $\Phi \in \mathcal{O}_{1}$ then $\Phi z_{1}=t_{1} \Phi$ and $\Phi w_{1}=t_{2} \Phi$. Note that, for any $j \in\{1, \ldots, k\}$, there exists $\Phi \in \mathcal{O}_{j}$ such that $\Phi z_{j}=t_{1} \Phi$ and $\Phi w_{j}=t_{2} \Phi$. In general, any flag $\Phi \in \mathcal{O}_{j}$ satisfies that $\Phi z_{j}=\bar{t}_{1} \Phi$ and $\Phi w_{j}=\bar{t}_{2} \Phi$ for some translations $\bar{t}_{1}$ and $\bar{t}_{2}$ with respect to the images of the translation vectors of $t_{1}$ and $t_{2}$ under some symmetry of $\mathcal{A}$.

Let $M$ be the maximum of the lengths of the translation vectors $t_{1}$ and $t_{2}$. Then every pair of points of the parallelogram determined by $\bar{t}_{1}$ and $\bar{t}_{2}$ have a distance less than $2 M$ between them. We claim that, given any element $x \in \operatorname{Mon}(\mathcal{A})$, there exists an element $y \in\left\langle z_{j}, w_{j} \mid j \in\{1, \ldots, k\}\right\rangle$ such that, for any flag $\Phi$ of $\mathcal{A}$, the (Euclidean) distance between the vertices of $\mathcal{A}$ in $\Phi$ and $\Phi x y$ is less than $2 M$. In fact, if for some flag orbit $j_{0}$ it happens that the distance between the vertices of $\Phi$ and $\Phi x$ is greater or equal than $2 M$, then there exist appropriate powers $\alpha_{1}$ and $\alpha_{2}$ of the translations $\bar{t}_{1}$ and $\bar{t}_{2}$ defined above, such that the distance between the vertices of $\Phi$ and $\bar{t}_{1}^{\alpha_{1}} \bar{t}_{2}^{\alpha_{2}} \Phi x$ is less than $2 M$ (it is enough to express in terms of $\bar{t}_{1}$ and $\bar{t}_{2}$ the translation mapping the vertex of $\Phi x$ to the parallelogram determined by the vertex of $\Phi$ together with the translations $\bar{t}_{1}$ and $\bar{t}_{2}$ ). By multiplying $x$ by the corresponding powers of $z_{j_{0}}$ and $w_{j_{0}}$ we obtain an element $x z_{j_{0}}^{\alpha_{1}} z_{j_{0}}^{\alpha_{2}}$ with the property that the distance between the vertices of $\Phi$ and $\Phi x z_{j_{0}}^{\alpha_{1}} z_{j_{0}}^{\alpha_{2}}$ is less than $2 M$. This argument can be applied to all flag orbits.

Recall that any element of $\operatorname{Mon}(\mathcal{A})$ is a permutation of the flags of $\mathcal{A}$. It follows from (2) that if $\Psi=\beta \Phi$ for some $\beta \in \Gamma(\mathcal{A})$ then the image of $\Psi$ under a given element $s \in \operatorname{Mon}(\mathcal{A})$ is determined by $\beta$ and the image of $\Phi$ under $s$. This implies that to describe an element in $\operatorname{Mon}(\mathcal{A})$ it suffices to define its action on only one flag of each flag orbit of $\mathcal{A}$ under $\Gamma(\mathcal{A})$.

Since there are finitely many flags whose vertices are at distance less than $2 M$ from the vertex of $\Phi$, there are finitely many cosets of $\left\langle z_{j}, w_{j} \mid j \in\{1, \ldots, k\}\right\rangle$ in $\operatorname{Mon}(\mathcal{A})$. Hence $\left\langle z_{j}, w_{j} \mid j \in\{1, \ldots, k\}\right\rangle$ has finite index in $\operatorname{Mon}(\mathcal{A})$.

Lemma 5. Let $\mathcal{A}, z_{j}$, and $w_{j}$ be as in Lemma 2. Then the monodromy elements $z_{j}$ and $w_{j}$ can be chosen so that $\left\langle z_{j}, w_{j} \mid j \in\{1, \ldots, k\}\right\rangle$ is a normal subgroup of $\operatorname{Mon}(\mathcal{A})$.

Proof. Let $C l(z)$ denote the normal closure of $z$ in $\operatorname{Mon}(\mathcal{A})$, and let $z_{j}$ and $w_{j}$ be as in the proofs of Lemmas 1 and 2. Since each $z_{j}$ and $w_{j}$ is a conjugate of $z_{1}$ it follows that $\left\langle z_{j}, w_{j} \mid j \in\{1, \ldots, k\}\right\rangle \leqslant C l\left(z_{1}\right)$. 
On the other hand, every conjugate of $z_{1}$ acts like $i d$ on all orbits of flags but one. Let $x \in \operatorname{Mon}(\mathcal{A})$ and let $\mathcal{O}_{j}$ be the flag orbit containing flags where $x^{-1} z_{1} x$ does not act like $i d$. Then $x^{-1} z_{1} x$ acts on any flag in $\mathcal{O}_{j}$ like a translation. From the actions of $x^{-1} z_{1} x$, $z_{j}$, and $z_{w}$ on the flags of each Archimedean tessellation $\mathcal{A}$, it can be seen that $x^{-1} z_{1} x$ is in the group $\left\langle z_{j}, w_{j}\right\rangle$. This shows that $\left\langle z_{j}, w_{j} \mid j \in\{1, \ldots, k\}\right\rangle \geqslant C l\left(z_{1}\right)$ and the lemma holds.

In what follows we derive a procedure to obtain a presentation in terms of generators and relations of $\operatorname{Mon}(\mathcal{A})$, for any given Archimedean tiling $\mathcal{A}$. We first define a finite map that arises from the quotient of $\operatorname{Mon}(\mathcal{A})$ by the normal subgroup $\left\langle z_{j}, w_{j} \mid j \in\{1, \ldots, k\}\right\rangle$ as in Lemma 5. The following lemma shows that such a map is in fact a toroidal quotient of $\mathcal{A}$.

Lemma 6. Let $\mathcal{A}$ be an Archimedean tiling of $\mathbb{E}^{2}$, let $z_{j}, w_{j}$ be as in Lemma 2, chosen to satisfy the conditions in Lemma 5 , and let $t_{1}, t_{2}$ be the translations in $\Gamma(\mathcal{A})$ such that $t_{1} \Phi=\Phi z_{1}$ and $t_{2} \Phi=\Phi w_{1}$ for some flag $\Phi$. Then

$$
\operatorname{Mon}(\mathcal{A}) /\left\langle z_{j}, w_{j} \mid j \in\{1, \ldots, k\}\right\rangle \cong \operatorname{Mon}\left(\mathcal{A} /\left\langle t_{1}, t_{2}\right\rangle\right) .
$$

Proof. Since there is a rank and adjacency preserving function from the flags of $\mathcal{A}$ to the flags $\mathcal{A} /\left\langle t_{1}, t_{2}\right\rangle$ it follows that there is a surjective group homomorphism from $\operatorname{Mon}(\mathcal{A})$ to $\operatorname{Mon}\left(\mathcal{A} /\left\langle t_{1}, t_{2}\right\rangle\right)$ (see [MPW] where these functions are called rap-maps). The kernel $H$ of such homomorphism contains $\left\langle z_{j}, w_{j} \mid j \in\{1, \ldots, k\}\right\rangle$ since all the monodromy elements in the latter preserve all flags in $\mathcal{A} /\left\langle t_{1}, t_{2}\right\rangle$. On the other hand, the action of every element in $H$ equals the action of some product of generators $z_{j}$ and $w_{j}$ implying that $H=\left\langle z_{j}, w_{j} \mid j \in\{1, \ldots, k\}\right\rangle$.

Note that the map $\mathcal{A} /\left\langle t_{1}, t_{2}\right\rangle$ lies on the torus, and in particular, its monodromy group is finite. This allows us to compute a presentation of $\operatorname{Mon}\left(\mathcal{A} /\left\langle t_{1}, t_{2}\right\rangle\right)$ in terms of generators and relations. Assume that $p$ is the least common multiple of the co-degrees of the faces, and $q$ is the degree of the vertices of $\mathcal{A}$, let $\pi$ be the natural quotient from $[p, q]$ to $\operatorname{Mon}(\mathcal{A})$, and suppose that

$$
\operatorname{Mon}\left(\mathcal{A} /\left\langle t_{1}, t_{2}\right\rangle\right)=[p, q] / C l_{[p, q]}\left(\left\{g_{1}, \ldots, g_{m}\right\}\right)
$$

for some $g_{i} \in[p, q]$, where $C l_{\Lambda}(X)$ denotes the normal closure of $X$ in the group $\Lambda$. Then, for $i \in\{1, \ldots, m\}$ there exists $h_{i} \in\left\langle z_{j}, w_{j} \mid j \in\{1, \ldots, k\}\right\rangle$, such that

$$
\pi\left(g_{i}\right)=h_{i} .
$$

Here we are assuming that $h_{i}$ is presented as an expression in terms of $\left\{z_{j}, w_{j} \mid j \in\right.$ $\{1, \ldots, k\}\}$, whereas $\pi\left(g_{i}\right)=\tilde{g}_{i}$ has the same expression in terms of the generators $r_{0}, r_{1}, r_{2}$ of $\operatorname{Mon}(\mathcal{A})$ as $g_{i}$ does in terms of the generators $\rho_{0}, \rho_{1}, \rho_{2}$ of $[p, q]$.

It follows from Lemma 3 that $\operatorname{Mon}(\mathcal{A})$ satisfies the relations

$$
\begin{aligned}
z_{i} z_{j} & =z_{j} z_{i}, \\
w_{i} w_{j} & =w_{j} w_{i}, \\
z_{i} w_{j} & =w_{j} z_{i},
\end{aligned}
$$


for $i, j \in\{1, \ldots, k\}$.

From Lemma 5 we conclude that for $i \in\{0,1,2\}$ and for $j \in\{1, \ldots, k\}$ there exist a word $x_{i, j}$ and a word $y_{i, j}$ on the set $\left\{z_{l}, w_{l} \mid l \in\{1, \ldots, k\}\right\}$ such that

$$
\begin{aligned}
r_{i} z_{j} r_{i} & =x_{i, j} \\
r_{i} w_{j} r_{i} & =y_{i, j}
\end{aligned}
$$

Clearly $\operatorname{Mon}(\mathcal{A})$ satisfies (5), (6) and (7). The next theorem shows that these relations suffice to describe $\operatorname{Mon}(\mathcal{A})$ as a quotient of the Coxeter group $[p, q]$.

Theorem 7. Let $\mathcal{A}$ be an Archimedean tiling of $\mathbb{E}^{2}$ with vertices of degree $q$ such that the least common multiple of the co-degrees of its faces is $p$. Let $z_{j}$ and $w_{j}$ be as in Lemma 5. Then $\operatorname{Mon}(\mathcal{A})$ is the quotient of the Coxeter group $[p, q]$ by the extra relations given in (5), (6) and (7), where $r_{i}$ in (7) should be interpreted as the corresponding generator $\rho_{i}$ of $[p, q]$.

Proof. This is equivalent to proving that the relations on the $r_{i}$ given by (5), (6) and (7) form a sufficient set of relations to determine if two arbitrary words in $r_{0}, r_{1}$ and $r_{2}$ represent the same word in $\operatorname{Mon}(\mathcal{A})$.

Let $g_{\omega} \in \operatorname{Mon}(\mathcal{A})$ be represented by a word $\omega$ on the $r_{i}$, and let $\omega_{i}$ be representatives from each of the cosets of $\mathrm{Cl}_{[p, q]}\left(\left\{\tilde{g}_{1}, \ldots, \tilde{g}_{m}\right\}\right)$ in $\operatorname{Mon}(\mathcal{A})$. Lemma 5 implies that we can rewrite $g_{\omega}=w_{i} \beta$, where $\beta \in \operatorname{Cl}_{[p, q]}\left(\left\{\tilde{g}_{1}, \ldots, \tilde{g}_{m}\right\}\right)$. Thus $\beta=\beta_{1} \tilde{g}_{i_{1}} \beta_{1}^{-1} \beta_{2} \tilde{g}_{i_{2}} \beta_{2}^{-1} \cdots \beta_{n} \tilde{g}_{i_{n}} \beta_{n}^{-1}$ for some choice of $\beta_{i}$ words on the $r_{i}$ and $\tilde{g}_{i_{k}} \in\left\{\tilde{g}_{1}, \ldots, \tilde{g}_{m}\right\}$. By (5)

$$
g_{\omega}=\omega_{i} \beta_{1} h_{i_{1}} \beta_{1}^{-1} \beta_{2} h_{i_{2}} \beta_{2}^{-1} \cdots \beta_{n} h_{i_{n}} \beta_{n}^{-1}
$$

where $h_{i_{j}} \in\left\langle z_{j}, w_{j} \mid j \in\{1, \ldots, k\}\right\rangle$. By $(7), z_{j} r_{i}=r_{i} x_{i j}$ and $w_{j} r_{i}=r_{i} y_{i j}$. Since $x_{i j}, y_{i j} \in$ $\left\langle z_{j}, w_{j} \mid j \in\{1, \ldots, k\}\right\rangle$, repeated applications of these relations show that $\beta_{k} h_{k_{l}} \beta_{k}=$ $\beta_{k} \beta_{k}^{-1} \hat{h}_{k_{l}}=\hat{h}_{k_{l}}$, where $\hat{h}_{k_{l}} \in\left\langle z_{j}, w_{j} \mid j \in\{1, \ldots, k\}\right\rangle$. Thus $g_{\omega}=\omega_{i} \Pi_{i=1}^{n} \hat{h}_{i_{j}}=\omega_{i} t$ for some $t \in\left\langle z_{j}, w_{j} \mid j \in\{1, \ldots, k\}\right\rangle$. By the commutativity of the generators of $\left\langle z_{j}, w_{j}\right| j \in$ $\{1, \ldots, k\}\rangle$ obtained as a consequence of $(6), t$ may be represented uniquely as a product of the form $\prod_{i=1}^{k}\left(z_{i}^{p_{i}} w_{i}^{q_{i}}\right)$ for some choice of $p_{i}, q_{i} \in \mathbb{Z}$. Thus every element of $\operatorname{Mon}(\mathcal{A})$ has a unique presentation of the form $\omega_{i} \Pi_{j=1}^{k}\left(z_{j}^{p_{j}} w_{j}^{q_{j}}\right)$, completing the argument.

Remark 8. To conclude this section we note that the translation vectors in Table 1 were verified to be of minimal length possible. To do this we analyzed, with the help of GAP [GAP], the action of $\operatorname{Mon}(\mathcal{A})$ on the toroidal quotient of $\mathcal{A}$ determined by the translation vectors of $z_{\mathcal{O}}$ and $w_{\mathcal{O}}$, and noticed that no element in $\operatorname{Mon}(\mathcal{A})$ acts like a translation on flags with preimage in $\mathcal{O}$ while fixing the others.

\section{The Minimal Regular Cover of (3.4.6.4)}

In this section we demonstrate how the theoretical results of the previous section are used to compute the monodromy group of the (3.4.6.4) tiling $\mathcal{A}$. The group $\Gamma(\mathcal{A})$ acts with four orbits on the flags of $\mathcal{A}$, and thus, following Lemma 2, we define $z_{j}$ and $w_{j}$ 
for $j \in\{1, \ldots, 4\}$. To describe the action of these monodromy elements in terms of translations, we pick a basis $\left(b_{1}, b_{2}\right)$ (see Figure 2 for a representation of the four flag orbits and this basis).

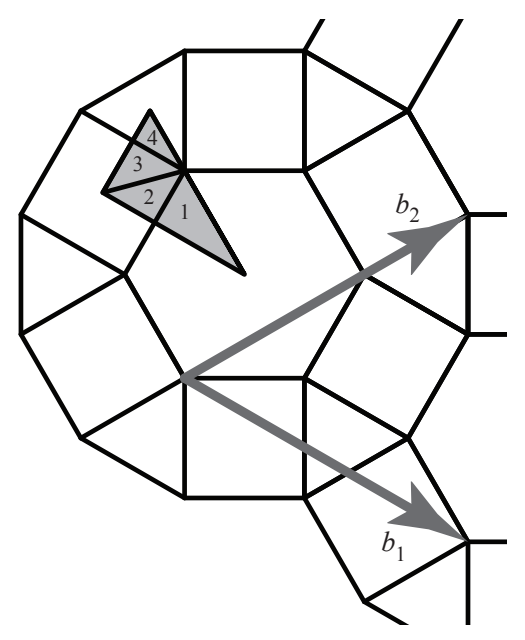

Figure 2: The four flag orbits and a basis for the translation subgroup of the symmetry group of (3.4.6.4).

Following Table 1 and Equation (4) we define:

$$
z_{1}:=\left(r_{0} r_{1}\right)^{3} r_{2}\left(r_{0} r_{1}\right)^{6} r_{2}\left(r_{1} r_{0}\right)^{3} r_{2}\left(r_{0} r_{1}\right)^{6} r_{2} \text { and } w_{1}:=r_{0} r_{1} z_{1} r_{1} r_{0} .
$$

Then, the proof of Lemma 2 shows how to construct the remaining $z_{j}$ and $w_{j}$. This yields:

$$
\begin{array}{ll}
z_{2}=r_{2} z_{1} r_{2} & w_{2}=r_{2} w_{1} r_{2} \\
z_{3}=r_{1} r_{2} z_{1} r_{2} r_{1} & w_{3}=r_{1} r_{2} w_{1} r_{2} r_{1} \\
z_{4}=r_{2} r_{1} r_{2} z_{1} r_{2} r_{1} r_{2} & w_{4}=r_{2} r_{1} r_{2} w_{1} r_{2} r_{1} r_{2}
\end{array}
$$

Let $\Phi$ be the shaded flag in Figure 2 that is in orbit 1 . The monodromy elements $z_{1}$ and $w_{1}$ act trivially on any flag not in the same orbit of $\Phi$ and act like translation on any flag in orbit 1. In particular, if $t_{1}=(2,0)$ and $t_{2}=(0,2)$; then $t_{1} \Phi=\Phi z_{1}$ and $t_{2} \Phi=\Phi w_{1}$. Furthermore, each $z_{j}$ and $w_{j}$ is a conjugate of $z_{1}$, and thus the conditions in Lemma 5 are satisfied. Therefore, by Lemma 6 ,

$$
\operatorname{Mon}(\mathcal{A}) /\left\langle z_{j}, w_{j} \mid j \in\{1, \ldots, k\}\right\rangle \cong \operatorname{Mon}\left(\mathcal{A} /\left\langle t_{1}, t_{2}\right\rangle\right) .
$$

In this case, the quotient $\mathcal{A} /\left\langle t_{1}, t_{2}\right\rangle$ consists of four hexagons, twelve squares, and eight triangles on the torus, and therefore has 192 flags. Thus, the group $\operatorname{Mon}\left(\mathcal{A} /\left\langle t_{1}, t_{2}\right\rangle\right)$ can be constructed as a subgroup of the symmetric group $S_{192}$. Then, a presentation for the group can be computed in GAP.

The result of this calculation is that $\operatorname{Mon}\left(\mathcal{A} /\left\langle t_{1}, t_{2}\right\rangle\right)$ is the quotient of the Coxeter group $[12,4]$ by the relators: 


$$
\begin{gathered}
g_{1}=\left(\rho_{2}\left(\rho_{0} \rho_{1}\right)^{4} \rho_{2} \rho_{1}\left(\rho_{0} \rho_{1}\right)^{3}\right)^{2} \\
g_{2}=\rho_{2}\left(\rho_{0} \rho_{1}\right)^{5} \rho_{2} \rho_{1} \rho_{2}\left(\rho_{0} \rho_{1}\right)^{3} \rho_{2}\left(\rho_{0} \rho_{1}\right)^{2} \rho_{2}\left(\rho_{0} \rho_{1}\right)^{4} \rho_{2} \rho_{1}, \\
g_{3}=\left(\rho_{1} \rho_{2}\left(\rho_{1} \rho_{0}\right)^{3} \rho_{2}\left(\rho_{1} \rho_{0}\right)^{5}\right)^{2} .
\end{gathered}
$$

Following Theorem 7, the monodromy group of $\mathcal{A}$ is the quotient of the Coxeter group $[12,4]$ by extra relations of types (5), (6) and (7). To determine the $h_{i}$ for the relations of type (5), we must determine the action of $g_{i}$ on the flags of $\mathcal{A}$. The result of this computation is that:

$$
\begin{gathered}
g_{1}=I d, \\
g_{2}=z_{3}^{-1} w_{3}, \\
g_{3}=z_{3} .
\end{gathered}
$$

The twenty eight relations of type (6) are trivial to write down, and all that remains to find a complete presentation for the monodromy group is to calculate the $x_{i, j}$ and $y_{i, j}$ for the relations of type (7). This is straight forward, and the result is shown in the following table. Each entry in the table is the result of conjugating the element of a column label by the element of the row label.

\begin{tabular}{|c||c|c|c|c|c|c|c|c|}
\hline & $z_{1}$ & $w_{1}$ & $z_{2}$ & $w_{2}$ & $z_{3}$ & $w_{3}$ & $z_{4}$ & $w_{4}$ \\
\hline \hline$r_{0}$ & $z_{1}$ & $w_{1}^{-1} z_{1}$ & $z_{2}$ & $w_{2}^{-1} z_{2}$ & $z_{3}^{-1}$ & $z_{3}^{-1} w_{3}$ & $z_{4}^{-1}$ & $w_{4} z_{4}^{-1}$ \\
\hline$r_{1}$ & $z_{1} w_{1}^{-1}$ & $w_{1}^{-1}$ & $z_{3}$ & $w_{3}$ & $z_{2}$ & $w_{2}$ & $w_{4}^{-1} z_{4}$ & $w_{4}^{-1}$ \\
\hline$r_{2}$ & $z_{2}$ & $w_{2}$ & $z_{1}$ & $w_{1}$ & $z_{4}$ & $w_{4}$ & $z_{3}$ & $w_{3}$ \\
\hline
\end{tabular}

Table 2: The monodromy element in the table is equal to the element obtained by conjugating the column label by the row label.

The monodromy group of the (3.4.6.4) tiling is obtained from the Coxeter group $[12,4]$ by adding the 3 relations of type (5), the 28 relations of type (6), and the 24 relations of type (7). However, many of these relations are redundant. Using GAP, we were able to reduce this list of 55 extra relations down to a set of 20. Our final presentation for $\operatorname{Mon}(\mathcal{A})$ can be found in Appendix A. We include descriptions of the sets of necessary relations for the remaining tilings as Appendix B. Unlike for the (3.4.6.4) tiling, the relations of type (5) are generally quite long and are instead found online at http://hdl.handle.net/11122/1232.

\section{Covers of the Archimedean Toroids}

All vertex-transitive maps on the torus are toroidal quotients of regular or Archimedean tessellations of $\mathbb{E}^{2}$ [PW11a], but not all such quotients are vertex-transitive. The minimal regular covers of (not necessarily vertex-transitive) toroidal maps obtained as toroidal quotients of the regular tessellations of $\mathbb{E}^{2}$ were found in [DM13]. All such covers are 
toroidal maps with Schlafli type $\{3,6\},\{4,4\}$, or $\{6,3\}$. In this section we discuss an approach to finding the minimal regular covers of toroidal quotients of the Archimedean tilings using the minimal regular covers of these tilings. For some of the toroidal quotients we explicitly describe how to obtain the minimal regular cover.

Let $\mathcal{A}$ be an Archimedean tiling. The elements $z_{\mathcal{O}}$ as in Table 1 and $w_{\mathcal{O}}$ as in (4) in $\operatorname{Mon}(\mathcal{A})$ act on flags in all but one flag orbit (namely $\mathcal{O}$ ) as $i d$, whereas they act on a given flag $\Phi \in \mathcal{O}$ like translations $t_{1}$ and $t_{2}$. Moreover, the translation vectors in Table 1 were chosen so that their norm is minimal possible (see Remark 8). It is easy to see, then, that if $x \in \operatorname{Mon}(\mathcal{A})$ acts on all flags not in $\mathcal{O}$ like $i d$ and acts on $\Phi$ like a translation $t_{3}$ then $t_{3} \in\left\langle t_{1}, t_{2}\right\rangle$.

Lemmas $3,4,5$, and 6 hold if we substitute $z_{\mathcal{O}}$ by $z_{\mathcal{O}}^{s}$ for some integer $s \geqslant 2$ while defining $w_{\mathcal{O}}$ as in (4) and $z_{i}, w_{i}$ as in the proof of Lemma 2 . In other words, given a normal finite index subgroup $H$ of $\operatorname{Mon}(\mathcal{A})$ isomorphic to $\mathbb{Z}^{2 k}=\left\langle y_{1}, \ldots, y_{2 k}\right\rangle$ as in Section 3 , the subgroup of $H$ isomorphic to $\left\langle s y_{1}, \ldots, s y_{2 k}\right\rangle$ is also normal and has finite index. Moreover,

$$
\operatorname{Mon}(\mathcal{A}) /\left\langle z_{j}^{s}, w_{j}^{s} \mid j \in\{1, \ldots, k\}\right\rangle \cong \operatorname{Mon}\left(\mathcal{A} /\left\langle t_{1}^{s}, t_{2}^{s}\right\rangle\right)
$$

if $z_{j}$ and $w_{j}$ are as in Lemma 6. The next theorem follows from (8).

Theorem 9. Let $\mathcal{A}$ be an Archimedean tiling of $\mathbb{E}^{2}$. Let $z_{\mathcal{O}}$ and $w_{\mathcal{O}}$ be as in Table 1 and (4) respectively, let $t_{1}$ and $t_{2}$ be translations such that $t_{1} \Phi=\Phi z_{\mathcal{O}}$ and $t_{2} \Phi=\Phi w_{\mathcal{O}}$ for some flag $\Phi$, and let $z_{i}$ and $w_{i}$ be as in the proof of Lemma 2. Let $\mathcal{A}_{t_{1}^{s}}, t_{2}^{s}$ be the toroidal map obtained from $\mathcal{A}$ by taking quotient by the translation group $\left\langle t_{1}^{s}, t_{2}^{s}\right\rangle$. Then $\operatorname{Mon}\left(\mathcal{A}_{t_{1}^{s}, t_{2}^{s}}\right)$ is obtained from $\operatorname{Mon}(\mathcal{A})$ by imposing the single extra relation $z_{\mathcal{O}}^{s}=i d$.

As an example, consider the map $\mathcal{M}=\mathcal{A}_{t_{1}^{2}, t_{2}^{2}}$ where $\mathcal{A}$ is the tiling 3.4.6.4. Then, by Theorem 9 we know that the minimal regular cover of $\mathcal{M}$ is obtained as the quotient of the Coxeter group $[12,4]$ by the elements in the table of Appendix A plus the single extra relation $z_{1}^{2}=i d$.

We note that if $\mathcal{A}_{t_{1}, t_{2}}$ is as in Theorem 9 then Relations (6) and (7) become redundant in $\operatorname{Mon}\left(\mathcal{A}_{t_{1}, t_{2}}\right)$ because of the extra relation $z_{\mathcal{O}}=i d$. This is consistent with what we knew, since the only defining relations of $\mathcal{A}_{t_{1}, t_{2}}$ are (5).

So far we have described the minimal regular covers of toroidal maps obtained as quotients of Archimedean tessellations by the specific translation groups $\left\langle t_{1}^{s}, t_{2}^{s}\right\rangle$. These particular quotients cover any other toroidal quotient of the Archimedean tessellations [DS]. Consequently, the minimal regular cover of a toroidal quotient $\mathcal{A}_{t_{1}^{s_{1}}} t_{2}^{r_{1}}, t_{1}^{s_{2}} t_{2}^{r_{2}}$ of an Archimedean tiling is a quotient of the minimal regular cover of one of the toroidal maps $\mathcal{A}_{t_{1}^{s}, t_{2}^{s}}$, and, given any particular such tiling, its cover can be found easily by computing the stabilizer of a flag of $\mathcal{A}_{t_{1}^{s_{1}} t_{2}^{r_{1}}, t_{1}^{s_{2}} t_{2}^{r_{2}}}$ under the action of the monodromy group of its cover. The determination of these convenient covers $\mathcal{A}_{t_{1}^{s}}, t_{2}^{s}$ for arbitrary toroidal quotients of Archimedean maps is beyond the scope of this paper, hence we do not present a full description of them. 


\section{Conclusions and Open Questions}

For each of the tilings described in this paper we have been able to identify a corresponding abelian extension of a finite group to which a minimal regular cover of the tiling may be associated. Critical to this construction are the existence of elements in the monodromy group of the tiling that act like translations in two different directions on one transitivity class of flags and fix all other classes. This is suggestive.

Conjecture 10. For each periodic tiling $\mathcal{T}$ of the plane there exist a pair of elements $w_{\mathcal{O}}, z_{\mathcal{O}} \in \operatorname{Mon}(\mathcal{T})$ with the property that $w_{\mathcal{O}}, z_{\mathcal{O}}$ act like translations in two different directions on all flags in one transitivity class of flags under the symmetry group of $\mathcal{T}$, and fix all flags in the other transitivity classes.

If the previous conjecture is true, the following conjecture would be a simple corollary by arguments similar to those presented in this paper.

Conjecture 11. Every periodic tiling of the plane admits a representation of the form given in Theorem 7 .

A broader question in the context of these results is the formulation of what it means for an infinite abstract polytope to be periodic in the absence of a geometric presentation (abstract polytopes are a generalization to higher ranks of abstract polyhedra, for more details see [MS02]). A first candidate would be to consider those polytopes of rank $d$ whose automorphism groups contain a free abelian subgroup of finite index of rank $(d-1)$, or translational periodic polytopes; in the case of the periodic tilings of the Euclidean plane the free abelian group is actually the group of translational symmetries of the tiling.

Given the context of the current work, a second candidate would be to consider those polytopes whose monodromy groups contain a free abelian subgroup of finite index of rank $(d-1) k$ where $d$ is the dimension (rank) of the abstract polytope, $k$ is the number of flag orbits under the automorphism group. This class of polytopes could be referred to as monodromy periodic. A natural additional requirement on the $(d-1) k$ generators is that, for each flag orbit $\mathcal{O}, d$ generators act nontrivially on flags in $\mathcal{O}$ while fixing all flags in the other orbits. Is every translational periodic polytope automatically a monodromy periodic polytope? Conversely, is every monodromy periodic polytope a translational periodic polytope? A contrasting approach to periodicity, in the context of aperiodic tilings, can be found in [Sch03], where a tiling of a Euclidean space by convex tiles is periodic if its automorphism group contains an element of infinite order.

The classification of automorphism groups of polytopes satisfying either of these definitions would be interesting since they would be freed of the geometric constraints of the crystallographic restriction, and so one might expect there to be more than the familiar 17 plane crystallographic groups, or the 230 crystallographic space groups. A related question about monodromy groups of abstract polytopes subject to the same restrictions is whether all such monodromy groups arise from Euclidean periodic tilings. 


\section{References}

[Bra27] H. R. Brahana, Regular maps and their groups, Amer. J. Math. 49 (1927), no. 2, $268-284$.

[CM80] H. S. M. Coxeter and W. O. J. Moser, Generators and relations for discrete groups, fourth ed., Ergebnisse der Mathematik und ihrer Grenzgebiete [Results in Mathematics and Related Areas], vol. 14, Springer-Verlag, Berlin, 1980.

[CPR+] Thierry Coulbois, Daniel Pellicer, Miguel Raggi, Camilo Ramírez, and Ferrán Valdez, The topology of the minimal regular cover of the archimedean tessellations, (in review).

[DM13] Kostiantyn Drach and Mark Mixer, Minimal covers of equivelar toroidal maps, (in review) (2013).

[DS] Kostiantyn Drach and Maksym Skoryk, Archimedean toroids and their minimal almost regular covers., (in preparation).

[GAP] M Schönert et al., Gap: Groups, algorithms and programming, Lehrstuhl D für Mathematik, Aachen (1994).

[GS87] Branko Grünbaum and G. C. Shephard, Tilings and patterns, W. H. Freeman and Company, New York, 1987.

[Har99a] Michael I. Hartley, All polytopes are quotients, and isomorphic polytopes are quotients by conjugate subgroups, Discrete Comput. Geom. 21 (1999), 289-298.

[Har99b] Michael I. Hartley, More on quotient polytopes, Aequationes Math. 57 (1999), $108-120$.

[HOW09] Isabel Hubard, Alen Orbanić, and A. Ivic Weiss, Monodromy groups and selfinvariance, Canad. J. Math. 61 (2009), 1300-1324.

[HW10] Michael I. Hartley and Gordon I. Williams, Representing the sporadic Archimedean polyhedra as abstract polytopes, Discrete Math. 310 (2010), no. 12, 1835-1844.

[MPW] Barry Monson, Daniel Pellicer, and Gordon I. Williams, Mixing and monodromy of abstract polytopes, Trans. of the AMS (in press).

[MS02] Peter McMullen and Egon Schulte, Abstract regular polytopes, Cambridge University Press, 2002.

[Orb07] Alen Orbanić, F-actions and parallel-product decomposition of reflexible maps, J. Algebraic Combin. 26 (2007), no. 4, 507-527.

[PW11a] Daniel Pellicer and Asia Ivić Weiss, Uniform maps on surfaces of non-negative Euler characteristic, Symmetry 22 (2011), no. 1-2, 159-196.

[PW11b] Daniel Pellicer and Gordon I. Williams, Quotient representations of uniform tilings, Symmetry Cult. Sci. 22 (2011), no. 3-4, 289-306.

[PW12] Daniel Pellicer and Gordon I. Williams, Minimal covers of the Archimedean tilings, part 1, Electron. J. Combin. 19 (2012), no. 3, \#P6. 
[Sch03] Egon Schulte, Combinatorial aperiodicity of polyhedral prototiles, in Discrete geometry, volume 253 of Monogr. Textbooks Pure Appl. Math., pages 397-406. Dekker, New York, 2003.

[Wil76] Stephen E. Wilson, New techniques for the construction of regular maps, Ph.D. thesis, University of Washington, 1976.

[Wi194] Stephen E. Wilson, Parallel products in groups and maps, J. Algebra 167 (1994), no. 3, 539-546.

[Wil02] Stephen E. Wilson, Families of regular graphs in regular maps, J. Combin. Theory Ser. B 85 (2002), no. 2, 269-289. 


\section{A Presentation for the monodromy group of (3.4.6.4)}

The monodromy group of the (3.4.6.4) tiling can be presented as the quotient of the Coxeter group $[12,4]$ by the following twenty additional relators.

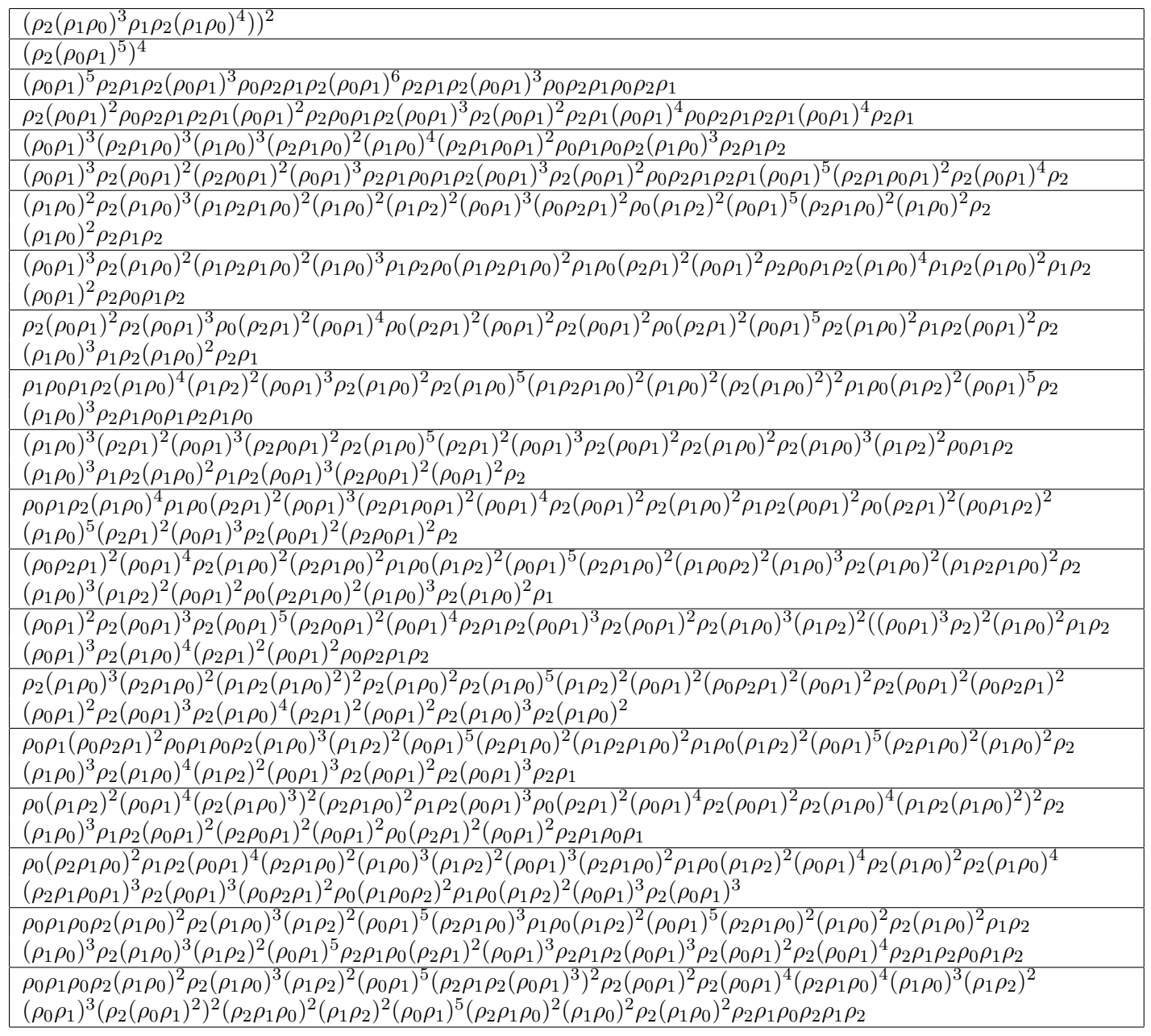

Table 3: Non-Coxeter relators for the presentation of Mon((3.4.6.4))

\section{B The remaining minimal regular covers}

In this section we give a summary of the relations in the presentations of the monodromy groups for the (3.3.4.3.4), (3.3.3.4.4), (4.6.12), and (3.3.3.3.6) tilings. We give these relations in terms of the monodromy elements $z_{j}$ and $w_{j}$ described in Lemma 2. Following Theorem 7, the equations (5), (6), and (7) define three different types of relations in the presentation. The relations of type (5) will be omitted here, as they are quite long. However, the full list of these relations can be found online at http://hdl.handle.net/11122/1232. Those of type (6) are trivial to write down and are omitted. In each of the tables below 
we summarize the relations of type (7), each monodromy element in the table is equal to the element obtained by conjugating the column label by the row label.

\section{B.1 (3.3.4.3.4)}

$$
\begin{array}{ll}
z_{1}=\left[\left(r_{1} r_{0}\right)^{3} r_{2} r_{1} r_{2}\right]^{2}\left[\left(r_{0} r_{1}\right)^{3} r_{2} r_{1} r_{2}\right]^{2} & w_{1}=r_{1} r_{0} z_{1} r_{0} r_{1} \\
z_{2}=r_{0} z_{1} r_{0} & w_{2}=r_{0} w_{1} r_{0} \\
z_{3}=r_{2} z_{1} r_{2} & w_{3}=r_{2} w_{1} r_{2} \\
z_{4}=r_{2} r_{0} z_{1} r_{0} r_{2} & w_{4}=r_{2} r_{0} w_{1} r_{0} r_{2} \\
z_{5}=r_{1} r_{2} r_{0} z_{1} r_{0} r_{2} r_{1} & w_{5}=r_{1} r_{2} r_{0} w_{1} r_{0} r_{2} r_{1}
\end{array}
$$

\begin{tabular}{|c||c|c|c|c|c|c|c|c|c|c|}
\hline & $z_{1}$ & $z_{2}$ & $z_{3}$ & $z_{4}$ & $z_{5}$ & $w_{1}$ & $w_{2}$ & $w_{3}$ & $w_{4}$ & $w_{5}$ \\
\hline \hline$r_{0}$ & $z_{2}$ & $z_{1}$ & $z_{4}$ & $z_{3}$ & $z_{5}^{-1}$ & $w_{2}$ & $w_{1}$ & $w_{4}$ & $w_{3}$ & $w_{5}$ \\
\hline$r_{1}$ & $w_{2}^{-1}$ & $w_{1}$ & $z_{3}^{-1}$ & $z_{5}$ & $z_{4}$ & $z_{2}$ & $z_{1}^{-1}$ & $w_{3}$ & $w_{5}$ & $w_{4}$ \\
\hline$r_{2}$ & $z_{3}$ & $z_{4}$ & $z_{1}$ & $z_{2}$ & $z_{5}$ & $w_{3}$ & $w_{4}$ & $w_{1}$ & $w_{2}$ & $w_{5}^{-1}$ \\
\hline
\end{tabular}

Table 4: The relations of type (7) for (3.3.4.3.4)

\section{B.2 (3.3.3.4.4)}

$$
\begin{array}{lll}
z_{1}=\left[\left(r_{0} r_{1}\right)^{3} r_{2}\left(r_{0} r_{1}\right)^{2} r_{2} r_{0} r_{1} r_{2}\left(r_{1} r_{0}\right)^{2} r_{2} r_{1} r_{2} r_{1} r_{2}\right]^{2} & w_{1}=r_{2} r_{1} r_{0} r_{1} r_{2} z_{1} r_{2} r_{1} r_{0} r_{1} r_{2} \\
z_{2}=r_{1} z_{1} r_{1} & w_{2}=r_{1} w_{1} r_{1} \\
z_{3}=r_{0} r_{1} z_{1} r_{1} r_{0} & w_{3}=r_{0} r_{1} w_{1} r_{1} r_{0} \\
z_{4}=r_{2} z_{1} r_{2} & w_{4}=r_{2} w_{1} r_{2} \\
z_{5}=r_{1} r_{2} z_{1} r_{2} r_{1} & w_{5}=r_{1} r_{2} w_{1} r_{2} r_{1}
\end{array}
$$

\begin{tabular}{|c||c|c|c|c|c|c|c|c|c|c|}
\hline & $z_{1}$ & $z_{2}$ & $z_{3}$ & $z_{4}$ & $z_{5}$ & $w_{1}$ & $w_{2}$ & $w_{3}$ & $w_{4}$ & $w_{5}$ \\
\hline \hline$r_{0}$ & $w_{1}^{-1}$ & $z_{3}$ & $z_{2}$ & $w_{4}^{-1}$ & $w_{5}$ & $z_{1}^{-1}$ & $w_{3}$ & $w_{2}$ & $z_{4}^{-1}$ & $z_{5}$ \\
\hline$r_{1}$ & $z_{2}$ & $z_{1}$ & $w_{3}^{-1}$ & $z_{5}$ & $z_{4}$ & $w_{2}$ & $w_{1}$ & $z_{3}^{-1}$ & $w_{5}$ & $w_{4}$ \\
\hline$r_{2}$ & $z_{4}$ & $z_{3}^{-1}$ & $z_{2}^{-1}$ & $z_{1}$ & $w_{5}^{-1}$ & $w_{4}$ & $w_{3}^{-1}$ & $w_{2}^{-1}$ & $w_{1}$ & $z_{5}^{-1}$ \\
\hline
\end{tabular}

Table 5: The relations of type (7) for (3.3.3.4.4)

\section{B.3 (4.6.12)}

$$
\begin{array}{ll}
z_{1}=\left(r_{0} r_{1}\right)^{4}\left[r_{2}\left(r_{0} r_{1}\right)^{-4}\right]^{2} r_{2}\left(r_{0} r_{1}\right)^{6} r_{2}\left[\left(r_{0} r_{1}\right)^{4} r_{2}\right]^{2}\left(r_{0} r_{1}\right)^{-4} r_{2}\left(r_{0} r_{1}\right)^{6} r_{2} & w_{1}=r_{1} r_{0} r_{1} z_{1} r_{1} r_{0} r_{1} \\
z_{2}=r_{1} z_{1} r_{1} & w_{2}=r_{1} w_{1} r_{1} \\
z_{3}=r_{2} z_{1} r_{2} & w_{3}=r_{2} w_{1} r_{2} \\
z_{4}=r_{1} r_{2} z_{1} r_{2} r_{1} & w_{4}=r_{1} r_{2} w_{1} r_{2} r_{1} \\
z_{5}=r_{2} r_{1} z_{1} r_{1} r_{2} & w_{5}=r_{2} r_{1} w_{1} r_{1} r_{2} \\
z_{6}=r_{2} r_{1} r_{2} z_{1} r_{2} r_{1} r_{2} & w_{6}=r_{2} r_{1} r_{2} w_{1} r_{2} r_{1} r_{2}
\end{array}
$$




\begin{tabular}{|c||c|c|c|c|c|c|c|c|c|c|c|c|}
\hline & $z_{1}$ & $z_{2}$ & $z_{3}$ & $z_{4}$ & $z_{5}$ & $z_{6}$ & $w_{1}$ & $w_{2}$ & $w_{3}$ & $w_{4}$ & $w_{5}$ & $w_{6}$ \\
\hline \hline$r_{0}$ & $z_{1}$ & $w_{2}$ & $z_{3}$ & $w_{4}^{-1}$ & $w_{5}$ & $w_{6}^{-1}$ & $z_{1}^{-1} w_{1}^{-1}$ & $z_{2}$ & $z_{3}^{-1} w_{3}^{-1}$ & $z_{4}^{-1}$ & $z_{5}$ & $z_{6}^{-1}$ \\
\hline$r_{1}$ & $z_{2}$ & $z_{1}$ & $z_{4}$ & $z_{3}$ & $z_{6}$ & $z_{5}$ & $w_{2}$ & $w_{1}$ & $w_{4}$ & $w_{3}$ & $w_{6}$ & $w_{5}$ \\
\hline$r_{2}$ & $z_{3}$ & $z_{5}$ & $z_{1}$ & $z_{6}$ & $z_{2}$ & $z_{4}$ & $w_{3}$ & $w_{5}$ & $w_{1}$ & $w_{6}$ & $w_{2}$ & $w_{4}$ \\
\hline
\end{tabular}

Table 6: The relations of type (7) for (4.6.12)

\section{B.4 (3.3.3.3.6)}

$$
\begin{array}{ll}
z_{1}=\left[\left(r_{0} r_{1}\right)^{3} r_{2} r_{1} r_{2} r_{0} r_{1} r_{2}\right]^{4} & w_{1}=r_{1} r_{0} z_{1} r_{0} r_{1} \\
z_{2}=r_{0} z_{1} r_{0} & w_{2}=r_{0} w_{1} r_{0} \\
z_{3}=r_{2} z_{1} r_{2} & w_{3}=r_{2} w_{1} r_{2} \\
z_{4}=r_{2} r_{0} z_{1} r_{0} r_{2} & w_{4}=r_{2} r_{0} w_{1} r_{0} r_{2} \\
z_{5}=r_{1} r_{2} z_{1} r_{2} r_{1} & w_{5}=r_{1} r_{2} w_{1} r_{2} r_{1} \\
z_{6}=r_{1} r_{2} r_{0} z_{1} r_{0} r_{2} r_{1} & w_{6}=r_{1} r_{2} r_{0} w_{1} r_{0} r_{2} r_{1} \\
z_{7}=r_{0} r_{1} r_{2} z_{1} r_{2} r_{1} r_{0} & w_{7}=r_{0} r_{1} r_{2} w_{1} r_{2} r_{1} r_{0} \\
z_{8}=r_{0} r_{1} r_{2} r_{0} z_{1} r_{0} r_{2} r_{1} r_{0} & w_{8}=r_{0} r_{1} r_{2} r_{0} w_{1} r_{0} r_{2} r_{1} r_{0} \\
z_{9}=r_{2} r_{1} r_{2} r_{0} z_{1} r_{0} r_{2} r_{1} r_{2} & w_{9}=r_{2} r_{1} r_{2} r_{0} w_{1} r_{0} r_{2} r_{1} r_{2} \\
z_{10}=r_{0} r_{2} r_{1} r_{2} r_{0} z_{1} r_{0} r_{2} r_{1} r_{2} r_{0} & w_{10}=r_{0} r_{2} r_{1} r_{2} r_{0} w_{1} r_{0} r_{2} r_{1} r_{2} r_{0}
\end{array}
$$

\begin{tabular}{|c||c|c|c|c|c|c|c|c|c|c|}
\hline & $z_{1}$ & $z_{2}$ & $z_{3}$ & $z_{4}$ & $z_{5}$ & $z_{6}$ & $z_{7}$ & $z_{8}$ & $z_{9}$ & $z_{10}$ \\
\hline \hline$r_{0}$ & $z_{2}$ & $z_{1}$ & $z_{4}$ & $z_{3}$ & $z_{7}$ & $z_{8}$ & $z_{5}$ & $z_{6}$ & $z_{10}$ & $z_{9}$ \\
\hline$r_{1}$ & $z_{2} w_{2}^{-1}$ & $w_{1}$ & $z_{5}$ & $z_{6}$ & $z_{3}$ & $z_{4}$ & $z_{8}$ & $z_{7}$ & $w_{10}^{-1}$ & $z_{9}^{-1} w_{9}$ \\
\hline$r_{2}$ & $z_{3}$ & $z_{4}$ & $z_{1}$ & $z_{2}$ & $z_{7}^{-1}$ & $z_{9}$ & $z_{5}^{-1}$ & $z_{10}$ & $z_{6}$ & $z_{8}$ \\
\hline
\end{tabular}

Table 7: Relations of type (7) for (3.3.3.3.6)

\begin{tabular}{|c||c|c|c|c|c|c|c|c|c|c|}
\hline & $w_{1}$ & $w_{2}$ & $w_{3}$ & $w_{4}$ & $w_{5}$ & $w_{6}$ & $w_{7}$ & $w_{8}$ & $w_{9}$ & $w_{10}$ \\
\hline \hline$r_{0}$ & $w_{2}$ & $w_{1}$ & $w_{4}$ & $w_{3}$ & $w_{7}$ & $w_{8}$ & $w_{5}$ & $w_{6}$ & $w_{10}$ & $w_{9}$ \\
\hline$r_{1}$ & $z_{2}$ & $z_{1}^{-1} w_{1}$ & $w_{5}$ & $w_{6}$ & $w_{3}$ & $w_{4}$ & $w_{8}$ & $w_{7}$ & $z_{10} w_{10}^{-1}$ & $z_{9}^{-1}$ \\
\hline$r_{2}$ & $w_{3}$ & $w_{4}$ & $w_{1}$ & $w_{2}$ & $w_{7}^{-1}$ & $w_{9}$ & $w_{5}^{-1}$ & $w_{10}$ & $w_{6}$ & $w_{8}$ \\
\hline
\end{tabular}

Table 8: Remaining relations of type (7) for (3.3.3.3.6) 